société qui ne souhait[ait] pas une diffusion massive de ce qui pou[v]ait menacer sa reproduction ». L. Le Van-Lemesle semble ainsi négliger le rôle joué par certaines fractions spécifiques " d'intellectuels de l'industrie ", ingénieurs et hauts fonctionnaires des services publics, dans la transformation de la fonction de cette discipline en véritable instrument de policy-making, justement durant les années qui précédèrent la Deuxième Guerre mondiale.

Toutefois, il est indiscutable que l'articulé et intelligent programme du Colloque a permis de neutraliser, avant même qu'il puisse prendre corps, le danger, lié à tout parcours réellement et sciemment innovateur, qui serait ici de remplacer l'idée d'un retard généralisé de l'enseignement supérieur français, face aux modèles étrangers, par celui de sa modernité à tout prix.

Giuliana Gemell.

\title{
ÉTUDES SUR SOREL
}

Cahiers Georges Sorel, 3, 1985.

Le numéro 3 des Cahiers Georges Sorel confirme les buts de la jeune Société d'études soréliennes, née des suites du Colloque tenu à l'École normale supérieure de la rue d'Ulm au printemps 1982 . En effet, si le colloque se présentait comme un état des études soréliennes dans le monde, la création de la Société correspondait au souhait de développer autour de la personne et de l'œuvre de Georges Sorel de nouvelles recherches. Il ne s'agissait ni de se livrer à une pure exégèse de l'œuvre de Sorel (chez qui, pourtant, les apparentes contradictions théoriques et la disparité des moyens d'expression justifient sans doute plus que chez beaucoup d'autres une lecture verticale), ni de tomber dans le travers d'une amicale sorélienne en publiant " les notes de blanchisseuses ", ni d'ouvrir la voie à d'éventuelles extrapolations d'ordre politique. La Société se donnait pour tâche de publier Sorel, dans le but de contribuer à une meilleure connaissance du monde intellectuel, socialiste et ouvrier du tournant du siècle.

L'cuvre du "solitaire de Boulogne " mérite attention à plusieurs titres : parce que s'y lisent les grands débats de la fin du $x_{1} x^{e}$ et du début du $x^{e}$ siècle, du syndicalisme révolutionnaire au fascisme, parce qu'elle est celle d'un ingénieur dont le destin personnel n'est pas indifférent, parce qu'elle est paradoxale et ambiguë. Peu lu ou mal lu, Sorel est porteur de lourdes postérités en mal de pères spirituels. La tentative des Cahiers est bien de fonder autour d'un penseur oublié ou masqué par ses héritiers une revue d'histoire intellectuelle, qui ne soit pas une pure histoire des idées. Et Sorel, au cœur d'un réseau européen de correspondants, du philosophe hégélien Croce au sociologue Roberto Michels, du socialiste "révisionniste " Bernstein à l'économiste Vilfredo 
Pareto, offre un remarquable point de mire. Les Cahiers pourraient être ainsi, dans un même domaine mais avec une autre optique, le complément du Mouvement social désormais plus spécifiquement consacré à l'histoire sociale "pure".

La pensée de Georges Sorel était l'objet privilégié des études et documents publiés dans les deux premiers numéros. Le numéro 3 renouvelle la problématique des Cahiers avec deux articles : celui de Christophe Prochasson "Sur l'environnement intellectuel de Georges Sorel : l'École des hautes études sociales ", celui de Michael Buckmiller " Georges Sorel et le " conservatisme révolutionnaire" en Allemagne ".

Si Sorel fut membre du Conseil d'administration de l'École des hautes études sociales, s'il y fit deux conférences, il la quitta pourtant en 1906, sans qu'on sache les raisons de ce départ ni quelle y fut précisément son activité. L'intérêt de l'article réside à la fois dans l'interprétation donnée de la participation de Sorel à l'administration de l'École et de son silence à ce sujet. Mais il est aussi, au-delà de Sorel, une étude sur la genèse de l'institutionnalisation des sciences sociales.

Quant à Michael Buckmiller, il propose une analyse de l'influence de Sorel dans l'Allemagne de l'après-guerre en des termes très pertinents qui permettent de dégager le concept flou d'influence ${ }^{1}$ : les lectures de Sorel par les théoriciens du « conservatisme révolutionnaire " allemand, Carl Schmitt, chez qui le mythe sorélien est vidé, d'après Buckmiller, de toute identité sociale pour ne garder qu'une fonction, Erwin von Beckerath, Eschman, Michael Freund, auteur de la première biographie intellectuelle de Sorel, et enfin Ernst Jünger. L'apport de l'article est de mettre à jour les mécanismes d'un processus qui, de lecture de Sorel en lecture de Sorel, a conduit à oublier l'œuvre au profit de son application. La théorie de Sorel, refondue à droite en un « conservatisme révolutionnaire " est devenue " un élément important pour l'intégration de l'intelligentsia de droite dans le fascisme ", " au point qu'à la fin l'œuvre de Sorel ne jouait plus aucun rôle ". Séduisante et savamment étayée, cette thèse ne manquera sans doute pas de contradicteurs. Malheureusement, le regard porté sur Sorel et sa postérité est encore loin de la neutralité.

Les deux autres articles s'inscrivent plus strictement dans la perspective des premiers Cahiers. Jacques Julliard, directeur de la revue, témoigne de sa fidélité à ses recherches récentes (La Faute à Rousseau, Paris, Le Seuil, 1985) en proposant un texte sur les rapports entre "Sorel, Rousseau et la Révolution française ". Pour Jacques Jullıard, l'opposition entre une culture dominante, " jacobine » et héritière de la Révolution, et une culture dominée « anti-rousseauiste " dont Sorel est un des fleurons, est une grille de lecture possible de l'histoire du socialisme. Sergio Romano, qui joint à la qualité de spécialiste de Sorel celle d'ambassadeur traite de : "Sorel et le système des relations internationales à la fin de la Première Guerre mondiale ", pièce à conviction dans le débat toujours ouvert du " nationalisme » de Sorel. La guerre créa un choc,

1. Voir aussi à ce sujet l'article de Chryssoula Kambas, «Walter Benjamin lecteur de Réflexions sur la violence», Cahiers Georges Sorel, 2, 1984. 
qui fut celui non pas de l'attitude attendue de dirigeants socialistes qu'il méprisait mais de la première faille dans la seule constante absolue de la pensée sorélienne : la foi dans le prolétariat. La haine de Sorel face à l'Union sacrée se nourrit aussi de l'espoir placé dans les nations " prolétaires" que sont pour lui l'Italie et l'Allemagne (espoir où Sergio Romano voit peut-être d'obscures raisons raciales).

La Société d'études soréliennes, éditrice des Cahiers, s'était aussi donné pour objectif la publication d'œuvres de Sorel. Son ambitieux projet d'œuvres complètes s'est sans doute heurté à des obstacles qui ont conduit à son ajournement. Mais la publication d'inédits dans les Cahiers témoigne, à un niveau plus modeste, de ce souci : la première lettre connue de Sorel (1872), lettres de Sorel à Einaudi, Michels, Croce, Bloch, à l'inverse des lettres de Bergson et Bernstein à Sorel dans les numéros 1 et 2 . Avec le numéro 3 commence une publication de plus longue haleine (puisqu'elle sera poursuivie sur quatre ans) des lettres de Sorel à Édouard Berth, son ami et disciple, avec lequel il entretenait des relations de confiance mutuelle. Édouard Berth, s'il ne fut jamais en désaccord avec Sorel et tout en épousant ses principales orientations, les accentua et suivit encore plus nettement que son aîné (il était de trente ans plus jeune que Sorel) la trajectoire qui mena ce dernier du syndicalisme révolutionnaire à l'Action française. Si l'on en croit l'intérêt de cette première partie (1904-1908), correspondant à la période "syndicaliste-révolutionnaire ", post-dreyfusienne de Sorel, ces lettres mériteraient une publication sous forme de volume, qui enrichirait le corpus des correspondances soréliennes parues jusqu'à ce jour. Pour ne citer que les plus importantes : les lettres à Delesalle, à Lagardelle, à Croce. Sorel, comme le rappelle Pierre Andreu dans son introduction, était loin d'être un partisan inconditionnel de la publication de correspondances privées. Il émit même de sérieuses réserves à propos de celle de Proudhon : " On ne devrait accueillir dans la correspondance imprimée d'un auteur célèbre que des pièces ayant au moins l'allure d'un très bon article de revue... je trouve particulièrement blâmable de livrer à la publicité les détails intimes de la vie privée... " (L'Indépendance, 15 février 1912). Mais quelqu'eût été l'avis de Sorel à cet égard, l'historien ne peut trouver dans ces lettres que matière à réflexion. C'est un document remarquable, non seulement sur un Sorel au quotidien (dans son rapport à son propre travail, ses réactions intellectuelles, ses difficultés d'écriture) dont la biographie de Pierre Andreu reste encore à ce jour le meilleur témoignage, mais aussi sur le fonctionnement des revues et les réseaux de relation intellectuels dans le monde socialiste du début du siècle.

Les Cahiers ne sont qu'une partie de l'effort accompli pour développer en France les études soréliennes. Les publications du livre de Shlomo Sand l'Illusion du politique et des Actes du colloque de 1982 (la bibliographie réalisée par Shlomo Sand pour les Cahiers, consacrée aux écrits sur Sorel est d'ailleurs complémentaire de celle élaborée dans le colloque par le même S. Sand et portant sur les écrits de Sorel) en sont la preuve.

Ainsi, outre l'apport essentiel à la connaissance de Georges Sorel et de son cuvre, les Cahiers peuvent trouver largement place dans le champ encore pau- 
vre des revues contemporaines consacrées aux problèmes intellectuels. L'histoire intellectuelle et celle des intellectuels en est à une étape importante de recherche de méthodes neuves, de symbiose entre les disciplines et entre les courants. L'étude du monde sorélien peut être le reflet de ces efforts. Elle pose aussi des problèmes plus spécifiques liés à l'histoire du mouvement ouvrier et socialiste où doivent se mêler étroitement histoire sociale, histoire économique, histoire des idées et des hommes sans tomber dans les travers de l'idéologie. Il est donc évident que l'orientation des Cahiers mérite une attention particulière et imposera des choix selon l'optique adoptée.

Françoise BLuM.

Shlomo SAnd, L'Illusion du politique. Georges Sorel et le débat intellectuel 1900. Paris, La Découverte, 1985. $13 \times 21,5,276$ p. («Armillaire »).

L'année 1985 aura-t-elle été à sa manière "l'année Sorel "? On pourrait bien le penser au vu du nombre de travaux importants parus ${ }^{1}$. Le livre de Shlomo Sand participe de cet intérêt renouvelé pour l'œuvre de Georges Sorel. Malgré un rétrécissement délibéré de son champ d'investigation, cantonné à la période des dix années (1893-1902) durant lesquelles Sorel dialogua sans cesse avec le marxisme, S. Sand a voulu dépasser l'étroitesse monographique. Son livre retiendra l'attention à trois niveaux : d'une part, à l'évidence, pour la biographie intellectuelle de Georges Sorel qu'on redécouvre ici, noyé qu'il était sous un déluge d'ouvrages à la rigueur scientifique parfois défaillante ${ }^{2}$; ensuite pour l'histoire intellectuelle en général, pour sa méthode et le sens d'une telle démarche mal représentée en France pour les XIXe et XX' $X^{e}$ siècles; le livre de Sand, enfin, permet de retrouver les grands débats intellectuels qui animèrent les milieux socialistes à la charnière des $\mathrm{XIX}^{\mathrm{e}}$ et $\mathrm{xx}^{\mathrm{e}}$ siècles. Cette mise en place de notre modernité à laquelle Sorel contribua n'est pas le moindre intérêt du livre de Shlomo Sand.

Georges Sorel est le type même de l'auteur à la réputation trouble : connu comme une des sources intellectuelles du fascisme ${ }^{3}$, pourfendu comme antisémite $^{4}$ ou comme partisan de l'Action française, méprisé comme révisionniste

1. Cahiers Georges Sorel, 2, 1984 et Cahiers Georges Sorel, 3, 1985 ; Actes du colloque Georges Sorel en son temps, Paris, Seuil, 1985, rassemblés par Jacques JULLIARD et Shlomo SAND; Cahier de l'Herne consacré à Georges Sorel, 1986.

2. Cf. le travail bibliographique réalisé par Shlomo Sand dans les numéros $l$ (1983) et 2 (1984) des Cahiers Georges Sorel dont il est un des principaux animateurs.

3. Cf. Michel Charzat, "Georges Sorel et le fascisme. Eléments d'explication d'une légende tenace ", Cahiers Georges Sorel, 1, 1983, p. 37-51. Grâce à une critique de sources serrée, Shlomo Sand fait aussi justice à Sorel de cette réputation au début de son ouvrage (p. 14-23).

4. Cf. Shlomo SAND, "Sorel, les Juifs et l'antisémitisme », Cahiers Geonges Sorel, 2, 1984, p. $7-36$. 
du marxismes, on sait moins que Sorel fut ardent dreyfusard pendant l'Affaire Dreyfus, ferme soutien des syndicalistes révolutionnaires en butte à la répression clemenciste, hostile à l'Union sacrée pendant la Première Guerre mondiale et favorable, jusqu'à sa mort en 1922, à la révolution bolchevique. $\mathrm{Ne}$ relèvera-t-on pas dans cet itinéraire quelque cohérence chez un auteur à qui l'on reprocha fréquemment ses contradictions?

Non que le livre de $\mathrm{S}$. Sand se plaise à soutenir le paradoxe qui ferait de Sorel un auteur limpide mais incompris. Sorel, écrivait Gramsci, « est compliqué, décousu, incohérent, superficiel, sibyllin ", mais l'originalité de ses points de vue, la force de son propos, en firent assurément l'égal des plus grands auteurs socialistes de cette fin du $\mathrm{xIX}^{\mathrm{e}}$ et de ce début du $\mathrm{xx}^{\mathrm{e}}$ siècle. Sorel méritait d'être relu.

Né en 1847, Georges Sorel fit ses études à l'École polytechnique puis à l'École des ponts et devint ingénieur de la fonction publique. Jusqu'en 1892, il ne fut qu'à l'origine de quelques publications qui révélaient un «philosophe libéral et conservateur ». C'est en 1893, sans que l'on sache d'ailleurs précisément pourquoi, que Sorel devint une " espèce de compagnon de route du parti guesdiste et des partis sociaux-démocrates européens ». Il prit alors connaissance de l'œuvre de Marx qu'il lut de façon originale tout en se considérant marxiste. Puis à partir de 1898 , sous l'influence des événements politiques, sociaux, culturels, pour l'essentiel cristallisés dans l'Affaire Dreyfus, Sorel se mue en critique du marxisme sans néanmoins y devenir hostile. Ce qu'il se mit surtout à charger fut ce qu'il considérait comme la caricature du marxisme, à savoir le discours tenu par la IIe Internationale dominée par la social-démocratie allemande.

C'est cette période de la vie de Sorel, au cours de laquelle le marxisme eut une place centrale, que Shlomo Sand a retenue. La volontaire limitation du sujet pourra laisser le lecteur sur sa faim dans la mesure même où le Sorel le plus « public » est celui des années ultérieures, le « théoricien des mythes et de la grève générale ", voire le "compagnon de route de l'Action française ". Cependant, comme le fait remarquer Shlomo Sand, l'appareil théorique sorélien se métamorphosant en permanence, il paraissait plus utile de se concentrer sur une courte période pour mieux saisir le mouvement d'une pensée aussi complexe. Sans négliger le fait que le problème soulevé par le livre n'est pas mince puisqu'il s'agit ni plus ni moins d'évoquer la rencontre d'un intellectuel français avec Marx et le marxisme et, plus largement, d'aborder la question, si mal étudiée encore ${ }^{6}$ de la diffusion du marxisme en France.

Or, sans conteste, Georges Sorel joua un rôle important dans ce processus, sans doute en raison de sa situation privilégiée le plaçant aux lisières du monde militant et du monde intellectuel. C'est lui qui, en 1902, fit le premier

5. Dans Pour Marx, Louis Althusser ironise sur les capacités philosophiques de Sorel : « L'Allemagne a eu Marx et Engels, et le premier Kautsky; la Pologne, Rosa Luxembourg; la Russie Plékhanov et Lénine ; l'Italie Labriola [...] (quand nous avions Sorel!) [...]" (Paris, Maspero, 1973, p. 14).

6. La seule synthèse existante est de Maurice DommangeT, L'Introduction du marxisme en France, Lausanne, Rencontre, 1969. 
exposé sur le matérialisme historique devant la très académique Société française de philosophie en présence des Bergson, Brunschvicg, Lachelier, Halévy, Lévy-Bruhl. On le voit aussi présent dans les plus " respectables » revues, Revue philosophique, Revue internationale de sociologie, etc.

Mais on le rematqua aussi parmi les animateurs actifs des petites revues militantes marxistes qui fleurirent en France dans les années 1890. En 1893, il commença à écrire dans la première revue marxiste française, l'Ère nouvelle. Puis de 1895 à 1897 , il se trouva au cœur de l'équipe du Devenir social qui diffusait alors la pensêe de Marx. Sorel est alors proche des guesdistes qui, pour le meilleur et pour le pire, étaient en ces années les seuls véritables médiateurs du marxisme en France. Les contacts théoriques que Sorel eut avec le marxisme (il connaissait mal l'allemand) se firent au travers de deux guesdistes : Paul Lafargue et Charles Bonnier, tous deux alors principaux traducteurs de Marx. Ce ne fut qu'après sa rupture avec Lafargue en 1897 que Sorel eut recours à Charles Andler, éminent germaniste, universitaire et socialiste anti-guesdiste.

Contrairement à beaucoup d'intellectuels français, Sorel aborda la lecture de Marx par Le Capital et non par Le Manifeste communiste, plus en quête d'une science que d'un manuel révolutionnaire. Sa lecture s'enrichit d'autres : celle des sociologues et de Durkheim en particulier, celle de Spencer (un " fumiste "), celle de Plekhanov, et surtout celle de Vico.

Ces contacts multiples l'amenèrent à une révision du marxisme orthodoxe sans que néanmoins, Sorel, jamais, n'appartînt à une école révisionniste. Dans une lettre à Croce, il se contente d'affirmer : " "Revenons à Marx", voilà ma devise. " Il garda toujours ses distances vis-à-vis de Bernstein, même s'il reconnaît en lui un " marxiste très authentique » qui pose de vraies questions, encore plus par rapport au révisionniste italien Merlino pour lequel pourtant il accepta, en 1898, de préfacer le livre Formes et essence du socialisme. Mais Sorel, par tempérament aussi, apprécie ces hommes dont l'attitude intellectuelle - celle qui les fait s'opposer à l'idéologie dominante de la IIe Internationale - l'attire. Sorel a l'indéniable goût de la marginalité et de la provocation intellectuelles.

Georges Sorel fut le contraire d'un esprit dogmatique. Son adhésion au marxisme se fit lucidement, sans complaisance. Il mit à plat ce qu'il considérait comme les failles de la pensée de Marx : la notion de dialectique, concept jugé trop flou et au demeurant plus présent dans la pensée d'Engels que dans celle de Marx (selon lui, il est nécessaire de dissocier les deux auteurs), la théorie de la valeur, la prétendue disparition des classes moyennes, etc., tous éléments qu'il faut peut-être, d'après Sorel, plus attribuer aux marxistes qu'à Marx lui-même.

La lecture que Sorel fit de Marx est bien autre chose que celle faite par l'orthodoxie marxiste, vulgate dogmatique dont la paternité reviendrait à Engels lui-même. Sorel, surtout à partir de l'Affaire Dreyfus, mettra en avant les aspects éthiques et juridiques, présents dans la lutte menée par le prolétariat et que, contrairement à ce que laissait penser la tradition, Marx avait bien vus. Ce sont les problèmes liés à l'acquisition d'une conscience de classe que 
Sorel retint aussi des travaux de Marx. Il s'opposa, enfin, violemment à la vision linéaire de l'histoire que proposaient les marxistes et qui, selon lui, était absente chez Marx. Lire Marx, sans les marxistes, contre les marxistes, telle était la gageure que s'était imposée Sorel.

En cela, les contacts intellectuels qu'il établit dans l'Europe entière l'aidèrent. En Italie surtout (où il écrivit dans de nombreuses revues), il noua plusieurs amitiés, pas toujours durables certes : Labriola, Croce, Colajanni, Pareto qui nourrit sa critique de l'économie marxiste. En Allemagne, où il entretint une correspondance avec Bernstein dont il ne reste malheureusement presque plus rien ainsi qu'avec Joseph Bloch, le directeur des Sozialistische Monatshefte' . En France, évidemment, où il trôna assidûment jusqu'en 1912 à la boutique des Cahiers de la Quinzaine que dirigeait Charles Péguy, mais où il correspondit aussi avec les auteurs les plus divers, de Gustave Le Bon (dès 1897) à Bergson.

Intellectuel Sorel ? Assurément, intellectuel pur même. Sa vie, ses amitiés le montrent. Aussi n'eut-on de cesse d'ironiser sur la critique qu'il fit du rôle des intellectuels dans le mouvement ouvrier! Mais pour Sorel, le fait est clair : l'intellectuel est en dehors du mouvement ouvrier, il n'a pas à y entrer par le biais de la fonction généreuse d'éducateur ( $L$ 'Avenir socialiste des syndicats qui constitue une des plus violentes critiques anti-intellectualistes de Sorel fut écrit au moment où les premières universités populaires apparaissaient). D'où le ralliement intellectuel de Sorel au syndicalisme révolutionnaire considéré comme seul véritable héritier de la pensée de Marx, d'où sa confiance dans le mouvement des Bourses du travail et, en général, dans les organisations ouvrières autonomes, à l'exclusion des partis socialistes accaparés par les "Intellectuels démocrates " tant haîs...

Le livre de Shlomo Sand s'achève sur un épilogue consacré au mythe chez Sorel, concept clé comme on le sait. On regrettera pourtant que cette dernière partie ne s'insère pas mieux dans le reste du livre et, qu'en conséquence, certaines redites apparaissent. Reste que Sand - et ce point constitue une nouveauté - s'est appliqué à présenter les rapports du marxisme de Sorel avec le concept de mythe qui s'oppose à l'illusion ou à l'utopie, pures créations de théoriciens et projections sur le futur. Le mythe constitue, quant à lui, un ciment susceptible d'unifier une classe dans le cadre d'une dynamique sociale. Ce n'est pas le mythe de la grève générale, bien connu de l'auteur des Réflexions sur la violence, que Sand présente mais celui de l'effondrement du capitalisme qui marque la clé de voûte de la politique marxiste.

Il faut donc souligner l'importance de l'ouvrage de Shlomo Sand, ouvrage difficile et peu accessible à ceux qui ne connaissent rien de Sorel, non seulement pour la nouveauté des éléments qu'il apporte mais aussi pour la méthode que son auteur a utilisée. Sand, en effet, a appliqué à Sorel la méthode que celui-ci avait utilisée dans sa lecture de Marx : se débarrasser d'abord de tous les propos parasitaires qui entourent une cuvre, la faussent, la trahissent. Revenir à Sorel, comme Sorel était revenu à Marx.

7. Cf. cette correspondance publiée et présentée par Pierre ANDreU et Michel Prat dans Cahiers Georges Sorel, 2, 1984, p. 107-129. 
Mais Shlomo Sand est aussi historien : il a la fibre du temps. Il fallait aussi remettre de l'ordre dans les écrits après les avoir rassemblés presque exhaustivement $^{8}$ (ce qui s'avérait particulièrement difficile pour un auteur aussi prolixe que Sorel!) afin de permettre une lecture synoptique indispensable à la compréhension de l'ensemble de l'œuvre de Sorel : de l'essai à l'article en passant par une correspondance si abondante et si plurielle...

Il fallait, enfin, éclairer tous ces textes par une mise en phase avec leur contexte, réflexe élémentaire à tout historien de l'histoire intellectuelle qui, plus que tout autre, affronte le texte à l'état " pur ". Shlomo Sand rend parfaitement compte et avec brièveté de tant d'aspects qui marquèrent la société dans laquelle vécut Sorel : état des forces politiques et sociales, débats au sein de la IIe Internationale, vie intellectuelle, évolution du mouvement ouvrier, etc. Son excellente connaissance de l'histoire de France dans toutes ses dimensions à la charnière des deux siècles aide le lecteur à se repérer dans un foisonnement de noms, d'événements et d'idées.

L'Illusion du politique est donc un livre exemplaire auquel on ne pourra guère que reprocher la dimension trop limitée de son objet. On souhaiterait une suite ou, pour mieux dire, un élargissement, un approfondissement. Il n'empêche que le livre de Shlomo Sand constitue une contribution majeure à l'histoire intellectuelle de la France au tournant des $\mathrm{XIX}^{\mathrm{e}}$ et $\mathrm{XX}^{\mathrm{e}}$ siècles, mais il doit aussi permettre (comme tous les bons ouvrages !) à d'autres chercheurs de suivre les pistes ouvertes.

Christophe Prochasson.

8. Cf. la bibliographie des œuvres de Sorel établie par Shlomo SAND dans Georges Sorel en son temps, op. cit. supra n. 1, p. 425-466.

Georges Sorel en son temps. Sous la dir. de Jacques Julliard et Shlomo SAND. Paris, Seuil, 1985. $14 \times 21,474$ p., index (" Philosophie politique »).

Après le livre de Shlomo Sand ${ }^{1}$, marquant une avancée sensible de la recherche sorélienne, viennent de paraître les Actes du Colloque consacré à Georges Sorel qui se tint au printemps 1982 dans les locaux symboliques de l'intellectualité - ceux de l'École normale supérieure - ce qui n'est pas un mince paradoxe lorsque l'on a à évoquer le cas d'un des ennemis les plus acharnés des intellectuels. Nouveau dossier donc à adjoindre aux déjà riches études soréliennes, qui profitent aussi de la sortie du numéro 3 des Cahiers Georges Sorel ${ }^{2}$ et de la parution d'un Cahier de l'Herne (1986).

Ce livre constitue avant tout un état des lieux. Il révèle une bonne part des acquis que la recherche a accumulés, en France et à l'étranger, dans les dernières années. Le contour idéologique de Sorel semble aujourd'hui mieux

1. Shlomo SAND, L'Illusion du politique. Georges Sorel et le débat intellectuel 1900, Paris, La Découverte, 1985.

2. S'adresser à la Société d'études soréliennes, 5, rue Las Cases, 75007 Paris. 
connu et les obscurités de sa pensée qui demeurent - voyez, par exemple, une question aussi rebattue que celle du mythe, d'ailleurs fort peu abordée dans les différentes contributions ici réunies - tiennent plus à leur auteur qu'à leurs exégètes ou à leurs analystes.

L'ouvrage ne rend compte, pour l'essentiel, que d'un Sorel idéologique, assez désincarné, parfois même éclaté et - on peut le regretter - insuffisamment situé dans l'histoire de son temps malgré les cinq premières contributions (Madeleine Rebérioux, Peter Schöttler, Zeev Sternhell, Maria Malatesta et Roberto Vivarelli) qui se sont essayées à ancrer Sorel dans son époque. La suite du livre pourrait nous les faire oublier.

Comme le signale Jacques Julliard dans une vigoureuse introduction, qui synthétise avec précision toutes les questions nourrissant une "actualité de Georges Sorel », ce furent par préférence les liens que l'auteur des Réflexions sur la violence entretint avec le marxisme qui furent abordés. Shlomo Sand, qui revient lui-même sur un des aspects de ce problème, certes essentiel, nous ayant déjà donné une étude qui peut passer pour définitive, les études du Sorel idéologique pourraient donner ici l'impression de piétiner... D'une part, en raison du fait que, si la pensée de Sorel tournait bien autour du marxisme de son temps, elle ne peut se réduire à celui-ci. D'autre part, parce que les études ici présentées souffrent d'un manque de repérages chronologiques, indispensables, comme l'a montré Shlomo Sand dans son livre, à la compréhension de Sorel. Le parachutage d'énoncés dans des contextes historiques différents, parfois éloignés de plusieurs années, faute commise y compris par les plus éminents spécialistes de l'œuvre de Georges Sorel, nuit à l'éclairage de celle-ci. Sans oublier l'actualisation parfois outrancière à laquelle donnent lieu certaines communications : établissement d'un parallèle entre d'éventuelles " erreurs " de Sorel et celles commises par les intellectuels des années 1960 et 1970 face à Mao Zédong, Castro, Che Guevara ou Hô Chi Minh (Georges Goriély), ou, mieux encore; rêve débridé d'un Sorel entendant Georges Marchais au Congrès du P.C.F. de février 1982 (Larry Portis)! Il est vrai que ce Colloque ne vit pas seulement la participation d'historiens mais aussi celle de sociologues, d'économistes et de politologues.

La troisième partie du livre, «Sorel et ses contemporains ", montre à quel point Sorel fut un intellectuel européen, en dialogue avec les plus grandes figures de son temps : Benedetto Croce (Sergio Romano), Emile Durkheim (Peter Nijhoff), Roberto Michels (Jean-Luc Pouthier), Giuseppe Prezzolini, le directeur d'une importante revue politique et littéraire de Florence, la Voce (Bruno Somalvico), Vilfredo Pareto (Giovanni Busino). Sorel fut assurément un intellectuel de son temps comme le montrent d'ailleurs aussi les nombreuses lectures qu'il faisait de tout ce qui paraissait, journaux, revues, livres. Reste à savoir peut-être ce que, de ce temps où se croisent encore les archaïsmes du XIXe siècle et la modernité du siècle suivant, Sorel a retenu. Une démarche intellectuelle à l'évidence résolument moderne, mais un contenu qui l'est peut-être moins, telle pourrait être la caractéristique de l'œuvre de Sorel.

De ces études de rencontres intellectuelles, on pourra se montrer déçu. D'une part, en raison de l'inégalité des plans intellectuels et historiques aux- 
quels se situent les différents personnages rencontrés par Sorel (que vient faire, par exemple, Prezzolini à côté d'un Croce ?). D'autre part, il s'agit, le plus souvent, de mises en parallèles idéologiques, qui, certes, se justifient théoriquement, plus que de rencontres intellectuelles, historiquement analysées, y compris dans leurs composantes purement amicales ou affectives qui ne manquaient pas chez Sorel. Peter Nijhoff préfère d'ailleurs parler de " confrontation du travail de Georges Sorel et de la sociologie d'Émile Durkheim ", les deux hommes n'ayant jamais correspondu et ne s'étant peut-être jamais rencontré. Les échanges entre intellectuels se résumeraient-ils à un échange d'idées? Nul ne le croira, même lorsqu'il s'agit de Georges Sorel qui se méfiait des notations privées comprises dans les correspondances ${ }^{3}$.

Jean-Luc Pouthier et Bruno Somalvico montrent, quant à eux, un autre versant de la correspondance sorélienne, plus privé, plus incarné. Si, assurément, un problème de sources existe pour saisir un "Sorel vivant " au travers de sa correspondance, il est regrettable que cet aspect n'ait pas au moins été signalé.

L'ouvrage s'achève sur un ensemble de quatre études consacrées à la diffusion du sorélisme, s'ouvrant par une étonnante géographie présentée par Robert Paris ; peu de pays échappent à son investigation : Italie qui réserva, comme chacun sait, bien meilleur accueil que la France à l'œuvre de Sorel, malgré cette trop célèbre lecture mussolinienne, Espagne, Portugal, Hongrie et même Amérique latine avec, en particulier, le cas, au Pérou, de Mariatégui. De leur côté, Jutta Scherrer pour la Russie, Larry Portis pour les pays anglosaxons et Michel Prat pour l'Allemagne rendent compte de la fortune de l'œuvre de Sorel hors de France où il fut lu avec parfois plus d'attention (seule l'Allemagne l'ignora vraiment).

Si la connaissance de l'œuvre de Sorel semble être aujourd'hui en bonne voie d'amélioration, il demeure tout un champ d'études encore presque vierge. Savoir ce qu'est devenue l'œuvre de Sorel sitôt celle-ci diffusée, étudier historiquement les problèmes de l'influence de Sorel sur les gauches comme sur les fascismes devraient aujourd'hui participer d'une réorientation du travail des chercheurs. Les contributions consacrées à la diffusion du sorélisme doivent pouvoir amorcer ce nouveau type de recherches.

Il est, enfin, un autre type d'études qu'il serait bon d'approfondir. Georges Sorel demeure un personnage méconnu, à la biographie mal saisie. Découvrir l'homme Sorel, le situer, par exemple, par rapport au corps des ingénieurs des Ponts de son temps, retracer plus précisément cet étrange itinéraire qui l'amena à rompre brutalement en 1892 avec sa carrière d'ingénieur, tout cela aidera, sans nul doute, à comprendre les linéaments de la littérature sorélienne.

Parce que occupant une place importante dans l'avant-garde idéologique de son temps, Georges Sorel n'a pas fini de passionner les chercheurs. Ses convergences-divergences avec tant d'intellectuels français et étrangers sont assez

3. Cf. l'introduction de Pierre Andreu aux lettres de Georges Sorel à Édouard Berth, Cahiers Georges Sorel, 3, 1985, p. 77-100. 
riches d'enseignements, qui contribuent à mieux connaitre la vie intellectuelle de son époque. Les Actes de ce Colloque le montrent avec force.

Il paraît temps que les études soréliennes, après les indéniables progrès accomplis depuis une dizaine d'années, doivent prendre un second souffle et ne pas se cantonner, ainsi que l'écrivait Jacques Julliard ${ }^{4}$, dans la publication tapageuse de quelques notes de blanchisseuse ou de correspondances abondantes mais sans intérêt véritable. Il semble que les animateurs des Cahiers Georges Sorel aient pris conscience de ce risque et souhaitent aujourd'hui ouvrir leur revue à des travaux dépassant le seul cas de leur auteur éponyme. On attend...

Christophe Prochasson.

\section{ART ET SOCIETÉ}

Claude-Gilbert Dubors, L'Imaginaire de la Renaissance. Paris, P.U.F., 1985. $14 \times 22,256$ p., bibliogr. ("Écriture ").

La multiplication récente des travaux et des enquêtes rendait utile et urgente une synthèse sur l'imaginaire de la Renaissance ; encore fallait-il avoir le courage de s'y lancer, et ce n'est sans doute pas le moindre mérite de ClaudeGilbert Dubois de l'avoir essayée. Ses recherches personnelles, de vastes lectures, un sens aigu de l'essentiel le prédisposaient certes à pareille entreprise. Il n'en reste pas moins que l'œuvre à accomplir se révélait immense et périlleuse, bien propre à s'attirer la pointilleuse sollicitude des critiques. Or, l'ouvrage qui en résulte, au-delà d'affirmations un peu trop tranchées et de tics d'écriture parfois agaçants, suscite (et suscitera encore longtemps, espérons-le) réflexions et discussions, ce qui est la meilleure preuve de sa fécondité. Ce compte rendu ne voudrait être qu'une de ces réflexions.

Il semble que la synthèse s'opère à deux niveaux. En effet, l'auteur enveloppe une approche thématique, qui détermine son plan, d'un effort de compréhension globale qui la dépasse et en utilise les conclusions partielles. En conséquence, bien que cette approche et cet effort s'entrelacent au fil du discours, ils ne reposent pas sur des bases identiques et ne procèdent pas exactement de la même démarche. Aussi convient-il de les distinguer.

Les approches thématiques portent à la fois sur les moyens et les méthodes de représentation (en particulier le langage et son utilisation) et sur les représentations elles-mêmes (celles de l'univers, du temps, du couple ville-jardin et

4. Présentation des Cahiers Georges Sorel, 1, 1983. 